\title{
Acción de la diminacina asociada al alopurinol y levamisol en hámsteres inoculados con Leishmania chagasi
}

\author{
Escalante, A.M.'; Burna, A.N.'2; Pérez-Gianeselli, M. ${ }^{3}$ \\ ${ }^{1}$ Becario de Posgrado, Secretaría Ciencia y Técnica Univ. Nac. Nordeste (UNNE), ${ }^{2}$ Cátedra Patología General, \\ ${ }^{3}$ Cátedra Farmacología, Facultad de Ciencias Veterinarias, UNNE, Cabral 2139, Corrientes (3400), Argentina. \\ E-mail: patgral@vet.unne.edu.ar
}

\begin{abstract}
Resumen
Escalante, A.M.; Burna, A.N.; Pérez-Gianeselli, M.: Acción de la diminacina asociada al alopurinol y levamisol en hámsteres inoculados con Leishmania chagasi. Rev. vet. 27: 2, 113-116, 2016. La leishmaniasis afecta a diferentes especies de animales domésticos y silvestres, siendo los perros su principal reservorio. En Corrientes (Argentina) se registra alta incidencia de leishmaniasis visceral canina, con riesgo para la salud pública. El objetivo del trabajo fue investigar la eficacia de la diminacina asociada al alopurinol y levamisol como tratamiento antileishmaniásico en hámsteres (Mesocricetus auratus). Se conformaron 3 grupos, cada uno con 26 hámsteres de dos meses de edad, ambos sexos y $60 \mathrm{~g}$ de peso. Cada grupo fue dividido en cuatro lotes, dos de 6 individuos cada uno y otros dos lotes de 7 individuos cada uno. Los animales de los lotes 1 y 3 de cada grupo fueron inoculados vía intraperitoneal ( $1 \mathrm{ml} / \mathrm{animal}$ ) con un macerado de bazo extraído de un canino naturalmente infectado con Leishmania chagasi. En el grupo 1, los animales del lote 3 fueron tratados con diminacina; en el grupo 2 (lote 3) fueron tratados con diminacina y alopurinol; y en el grupo 3 (lote 3) fueron tratados con diminacina, alopurinol y levamisol. A partir de los 70 días de comenzadas las inoculaciones, se realizaron eutanasias a dos animales de cada lote y grupo cada 30 días, de tal manera que pudieran investigarse animales con 30, 60 y 90 días de tratamiento. Los roedores infectados y medicados presentaron menor sintomatología y mejor estado general, evidenciado por la conservación del manto piloso, buena condición corporal, mantenimiento del apetito y conducta normal, en contraste con los animales inoculados sin tratamiento. Se concluye que la utilización aislada de diminacina (leishmanicida) produjo el mismo efecto protector (disminuyendo la sintomatología provocada por L. chagasi), que al estar asociada con alopurinol (leishmaniostatico) y levamisol (inmunomodulador), aunque ninguno de los tratamientos ensayados obtuvo la la curación parasitológica, ya que persistió la presencia de los parásitos.
\end{abstract}

Palabras clave: hamster, leishmaniasis, diminacina, alopurinol, levamisol.

\begin{abstract}
Escalante, A.M.; Burna, A.N.; Pérez-Gianeselli, M.: Action of the association diminazine-allopurinol-levamisole in hamsters inoculated with Leishmania chagasi. Rev. vet. 27: 2, 113-116, 2016. Leishmaniasis affects different species of domestic and wild animals, being the canines its main reservoir. In Corrientes (Argentina), a high incidence of canine visceral leishmaniasis is registered, with risk for the public health. The aim of this study was to demonstrate the in vivo effect of diminazine, associated with allopurinol and levamisole, as antileishmanial hamster treatment, in order to use these treatment as a therapeutic alternative for the control and possible eradication of leishmaniasis in dogs. Tree groups were formed with 26 hamsters (Mesocricetus auratus) two months old, both sexes, $60 \mathrm{~g}$ weight, which were divided into four lots, two batches of 6 individuals each, and two batches of 7 individuals each. Animals from lots 1 and 3 were inoculated intraperitoneally ( $1 \mathrm{ml} / \mathrm{animal})$ with a macerated spleen removed from a dog naturally infected with Leishmania chagasi. Lot 3 group 1 animals were treated with diminazine; group 2 lot 3 animals were treated with allopurinol and diminazine, and group 3 lot 3 were treated with diminazine, allopurinol and levamisole. Starting from 70 days of the beginning of inoculations, euthanasias were carried out on two animals from each batch and group every 30 days, so that the animals with 30, 60 and 90 days of treatment could be investigated. Medicated-infected rodents showed fewer symptoms and better overall welfare, as evidenced by their normal state of the hair
\end{abstract}


coat, good body condition, maintaining the eating habits and behavior, in contrast to the animals inoculated with L. chagasi without treatment. It is conclude that the isolated use of diminazine (leishmanicidal) produced the same protective effect (diminishing the symptomatology caused by $L$. chagasi) that when being associated with allopurinol (leishmaniostatic) and levamisol (immunomodulator), although none of the rehearsed treatments obtained the parasitological cure, since the presence of the parasites persisted.

Key words: hamster, leishmaniasis, diminazine, allopurinol, levamisole.

\section{INTRODUCCIÓN}

La leishmaniasis afecta a diferentes especies de animales domésticos y silvestres, siendo los caninos su principal reservorio ${ }^{4}$. En Corrientes (Argentina) tal parasitosis asume considerable importancia por su impacto en salud pública, debido a la alta incidencia de leishmaniasis visceral canina.

Empleando el hámster como modelo experimental de leishmaniasis visceral, otros autores reportaron la existencia de una pérdida de la respuesta inmune celular específica al antígeno, similar a la que ocurre en el ser humano ${ }^{13}$. Otros estudios han demostrado que la infección sistémica por Lishmania donovani en hámster resulta en un aumento progresivo de la carga parasitaria en hígado y bazo, provocando hepato y esplenomegalia, así como caquexia, pancitopenia y muerte ${ }^{11}$.

La diminacina es una droga con probada actividad (in vitro) inhibitoria del crecimiento de $L$. donova$n i$, mostrando mayor efecto a altas concentraciones ${ }^{9}$. También ha sido utilizada durante décadas para el tratamiento de tripanosomosis y babesiosis ${ }^{6}$. Su mecanismo de acción consiste en inhibir la síntesis de ADN y el metabolismo energético del parásito ${ }^{16}$.

El alopurinol se ha utilizado como coadyuvante e incluso como tratamiento único cuando no es posible el uso de sales antimoniales o de miltefosina. El alopurinol es una pirazolopirimidina utilizada como tratamiento de la gota y la litiasis úrica en seres humanos; es relativamente económico y se administra por vía oral, registrando muy pocos efectos secundarios (algunos casos de anemia, episodios breves de diarrea y cristaluria/urolitiasis de xantina). Su principal desventaja es que se trata de un fármaco parasitostático cuya única acción es frenar lentamente el crecimiento de Leishmania $s p$. El fármaco es un análogo de las bases púricas y su mecanismo de acción consiste en alterar la síntesis proteica de las leishmanias ${ }^{7}$.

Ciertos fármacos antiparasitarios han revelado poseer una actividad inmunomoduladora, entre ellos: levamisol, tiabendazol, fenvalerato, oxfendazol, fenbendazol, niridazol, metronidazol, carbofurano e ivermectina. Los efectos inmunomoduladores de estas drogas antiparasitarias, han sido estudiados en enfermedades bacterianas, virales, micóticas y helmínticas, así como en procesos neoplásicos y autoinmunes ${ }^{17}$.

El levamisol (6-fenil 2,3,5,6-tetrahidro imidazol [2,1-b] tiazol hidroclorato) es un fármaco representati- vo de dicho grupo de agentes, inicialmente desarrollados como antihelmínticos y usados extensamente para el tratamiento de vermes gastrointestinales y tisulares. Los estudios para demostrar su actividad inmunomoduladora han generado resultados contradictorios. El levamisol administrado en combinación con la vacuna de parvovirus canino, potencia la producción de anticuerpos, incrementa la actividad fagocítica y estimula la proliferación de linfocitos ${ }^{15}$. En la inmunidad celular, el efecto del levamisol es ejercido principalmente sobre linfocitos $\mathrm{T}$ anérgicos, macrófagos y leucocitos polimorfonucleares ${ }^{8}$.

El objetivo del presente trabajo fue demostrar el efecto in vivo de la asociación diminacina-alopurinollevamisol como tratamiento antileishmaniásico en hámsteres, con la finalidad de emplearla como alternativa terapéutica para el control y posible erradicación de tal enfermedad en caninos.

\section{MATERIAL Y MÉTODOS}

Se conformaron 3 grupos, cada uno con 26 hámsteres (Mesocricetus auratus) de dos meses de edad, ambos sexos y $60 \mathrm{~g}$ de peso, los cuales fueron divididos en cuatro lotes, dos lotes de 6 individuos cada uno y dos lotes de 7 individuos cada uno. Durante el tiempo que duró la experiencia se les administró agua y alimento ad libitum, observándose diariamente la eventual presentación de lesiones de piel, modificaciones de condición corporal y/o cambios de comportamiento.

Las tareas de observación y limpieza de las jaulas se realizaron diariamente en horario vespertino. Este procedimiento fue avalado por el Comité de Ética y Bioseguridad de la Facultad de Ciencias Veterinarias de la UNNE (Protocolo 0037). De acuerdo a la droga utilizada, los animales se agruparon de la siguiente manera:

\section{Grupo 1: diminacina}

Lote $1(\mathrm{n}=7)$ animales inoculados con L. chagasi.

Lote $2(\mathrm{n}=6)$ animales tratados con diminacina.

Lote $3(\mathrm{n}=7)$ animales inoculados con L. chagasi y tratados con diminacina.

Lote $4(\mathrm{n}=6)$ animales control.

\section{Grupo 2: diminacina y alopurinol}

Lote $1(\mathrm{n}=7)$ animales inoculados con L. chagasi. Lote $2(n=6)$ animales tratados con diminacina y alopurinol. 
Lote $3(\mathrm{n}=7)$ animales inoculados $\mathrm{c} /$ L. chagasi $\mathrm{y}$ tratados c/diminacina y alopurinol.

Lote $4(n=6)$ animales control.

\section{Grupo 3: diminacina, alopurinol y levamisol}

Lote $1(\mathrm{n}=7)$ animales inoculados con L. chagasi.

Lote $2(n=6)$ animales tratados con diminacina, alopurinol y levamisol.

Lote $3(\mathrm{n}=7)$ inoculados $\mathrm{c} /$ L. chagasi y tratados $\mathrm{c} /$ diminacina, alopurinol y levamisol.

Lote $4(n=6)$ animales control.

Los animales de los lotes 1 y 3 de cada grupo fueron inoculados vía intraperitoneal ( $1 \mathrm{ml} / \mathrm{animal})$ con un macerado de bazo extraído de un canino naturalmente infectado con L. chagasi (en solución fisiológica estéril a $37^{\circ} \mathrm{C}$ ), cuyo diagnóstico fue realizado mediante los métodos serológico y parasitológico directo.

Para confirmar la existencia de infección, luego de transcurridos 70 días de la inoculación ("día 0"), cada 30 días se llevaron a cabo eutanasias a dos animales de cada lote y grupo, de tal manera que pudieran investigarse animales con 30, 60 y 90 días de tratamiento. La eutanasia se efectuó con pentobarbital sódico y difenilhidantoina sódica a dosis de $0,3 \mathrm{mg} / \mathrm{kg}$, vía intraperitoneal. La confirmación de la infección se realizó por diagnostico parasitológico directo.

Confirmado el diagnóstico positivo comenzó la administración de los tratamientos programados. El tratamiento con diminacina para los 3 grupos, se efectuó por vía intramuscular profunda con una dosis de $3,5 \mathrm{mg} / \mathrm{kg}$ de peso, el mismo se realizó cada 15 días con una primera dosis que se administró 70 días post inoculación de los amastigotes de L. chagasi a todos los animales de los lotes 2 y 3 (tratados e infectados respectivamente) de cada grupo. El tratamiento con alopurinol a los lotes 2 y 3 de los grupos 2 y 3 , se aplicó a razón de $10 \mathrm{mg} / \mathrm{kg}$ cada 24 horas durante 30 días, vía oral. El tratamiento con levamisol a los lotes 2 y 3 se realizó a dosis de 2,5 a $5 \mathrm{mg} / \mathrm{kg}$ por vía subcutánea en un régimen de tres días de tratamiento, seguidos de cuatro días de descanso.

Se realizaron eutanasias a dos animales de cada lote y grupo cada 30 días, de tal manera que pudieran investigarse animales con 30, 60 y 90 días de tratamiento. Luego de cada eutanasia fue realizada la necropsia de los animales mediante la técnica convencional. Se realizaron improntas de bazo que fueron fijadas con alcohol de $96^{\circ}$, coloreadas con Giemsa y examinadas con microscopio óptico. Los órganos enteros (cerebro, corazón, pulmones, bazo, hígado, estómago, intestinos, genitales, riñones y piel) fueron inmediatamente fijados en formol bufferado al 10\%, durante 48 horas.

\section{RESULTADOS Y DISCUSIÓN}

Los roedores infectados y medicados presentaron menor sintomatología y mejor estado general, evidenciado por el buen estado del manto piloso, adecuada condición corporal, mantenimiento de los hábitos alimenticios y conducta normal. En contraste, los animales inoculados con L. chagasi sin administración de tratamiento (lote 1 de cada grupo), revelaron progresiva pérdida del estado corporal, adelgazamiento, debilidad, desprendimiento de pelo y lesiones ulcero-costrosas en la región nasal, en coincidencia con hallazgos de otros investigadores $2,5,10,16,18$. Tres hamsters murieron antes de los 160 días post-inoculación .

En el examen macroscópico de los órganos de los animales inoculados con el parásito, en todos los casos se advirtió hepato y esplenomegalia (Figura 1), mientras que en los lotes sin inocular no se registraron alteraciones en dichos órganos ${ }^{11}$.

En los frotis de bazo coloreados con Giemsa se visualizaron amastigotes de L. chagasi de localización extra e intracelular (citoplasma de macrófagos), tanto en los animales tratados como en los no tratados con diminacina, alopurinol y levamisol. Cada amastigote se identificó por la existencia de dos estructuras basófilas esféricas, una de mayor tamaño que la otra (núcleo y kinetoplasto del parásito), rodeadas por citoplasma claro de $2-5 \mu \mathrm{m}$ de diámetro.

Los animales de los lotes controles (lotes 4 de cada grupo, no tratados ni inoculados), no presentaron sintomatología alguna. Al examen macroscópico no se evidenciaron alteraciones morfológicas ni lesiones. Tampoco se visualizaron anomalías al examen microscópico.

La eficacia de la diminacina como antileishmaniásico ya había sido demostrada in vitro a través de cultivos celulares ${ }^{9}$. Según experiencias previas de los autores del presente trabajo, hámsteres inoculados con L. chagasi y tratados con diminacina revelaron efectos benéficos en la sintomatología clínica de la leishmaniasis visceral.

Existe un acotado número de drogas leishmanicidas en el mercado, las mismas son de uso humano y no

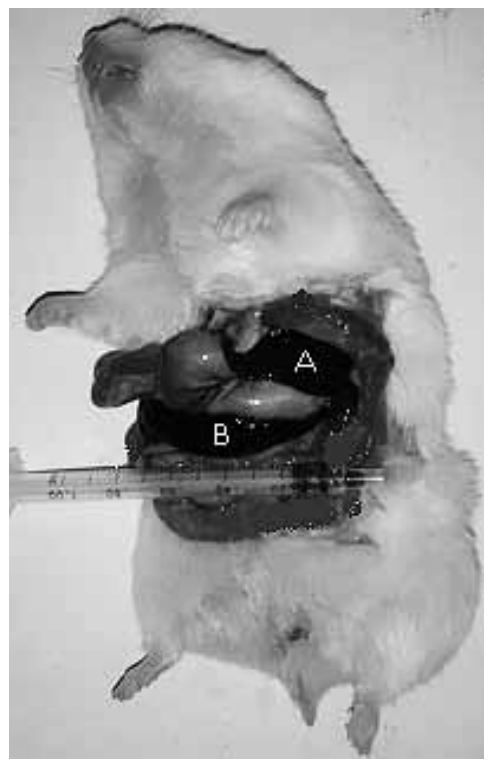

Figura 1. A: hepatomegalia, B: esplenomegalia. 
están permitidas para el tratamiento de caninos debido al riesgo que existe en generar resistencia del parásito a las mismas ${ }^{3}$. Hasta el momento, los trabajos realizados con alopurinol abordaron aspectos de la leishmaniasis humana, en tanto que la actividad inmunomoduladora del levamisol fue estudiada en enfermedades infecciosas y parasitosis helmínticas 7, 10,17.

Escasos estudios fueron realizados in vivo para investigar aspectos de la leishmaniasis visceral canina como los abordados en la presente investigación, donde se emplearon animales de laboratorio aplicando las dosis recomendadas por los laboratorios elaboradores ${ }^{14}$. Tanto el lote 3 del grupo 1 tratado con diminacina, el lote 3 del grupo 2 tratado con diminacina y alopurinol y el lote 3 del grupo 3 tratado con diminacina, alopurinol y levamisol, como los lotes 1 (control) de cada grupo (1, 2 y 3), inoculados con L. chagasi, presentaron amastigotes en las improntas de bazo ${ }^{1,11,12}$. Tal hallazgo fue independiente del tiempo transcurrido desde la inducción de la infección experimental.

En los grupos 2 y 3 , los animales inoculados y tratados con diminacina, alopurinol y levamisol mantuvieran clínicamente un buen estado de salud al igual que el grupo 1, sin evidenciar diferencias atribuibles al tipo de tratamiento utilizado. Ello podría sugerir que la droga que generó efectos benéficos en los animales infectados fue la diminacina. No se pudo ponderar el efecto de la asociación de fármacos sobre el aumento o disminución de las cargas parasitarias.

Debería concluirse proponiendo que la utilización exclusiva de diminacina como leishmanicida tendría el mismo efecto protector que asociada con alopurinol como leishmaniostático y levamisol como inmunomodulador. Tales fármacos podrían aminorar la sintomatología clínica de L. chagasi, pero no lograrían la curación porque persistió la presencia de los parásitos. Es menester continuar investigando la potencialidad de estas -y otras- drogas contra leishmanias, recurriendo a nuevos protocolos de dosis y tiempos, quizás monitoreados por técnicas de biología molecular como la reacción en cadena de la polimerasa (PCR cuantitativa), a efectos de encontrar un tratamiento efectivo contra la enfermedad.

Agradecimiento. Al Dr. Edgardo Borda, Director del Centro Nacional de Parasitología y Enfermedades Tropicales de la Universidad Nacional del Nordeste, por haber permitido llevar a cabo la experiencia en el bioterio de dicha institución.

\section{REFERENCIAS}

1. Alvar J, Cañavate C, Molina R, Moreno J, Nieto J. 2004. Canine leishmaniasis. Adv Parasit 57: 1-88.

2. Carrasco L, de Lara FC, Martín E. 1997. Acute haemorrhagic pancreatitis associated with canine visceral leishmaniasis. Vet Rec 141: 519-571.
3. Desjeux P. 1996. Leishmaniasis. Public health aspects and control. Clin Dermatol 14: 417-423.

4. Desjeux P. 2004. Leishmaniasis: current situation and new perspectives. Comp Immun Microb \& Infect Dis 27: 305-318.

5. Ferrer L, Juanola I, Ramos A, Ramis A. 1991. Chronic colitis dueto Leishmania infection in two dogs. Vet Pathol 28: 342-343.

6. Fussgänger R, Bauer F. 1962. Berenil, a new chemotherapeutic agent in veterinary medicine: data from Chemotherapeutic and Parasitological Laboratory of Farbwerke Hoechst. On line: http://www.jsava.co.za/index.php/jsava/ article/viewFile/513/492

7. Gómez N, Estevez O, Gisbert MA, Blanco A, Castillo V, Wolberg A. 2011. Leishmaniosis visceral en los caninos y felinos: actualización. Vet Arg 28: 282.

8. Guerrero J. 1977. Immunological modulating influence of levamisole. Proceedings of the Heartworm Symposium, Atlanta (Georgia), p.18-20.

9. Leishman WB. 1903. On the possibility of occurrence of tripanosomiasis in India. Br Med J 30: 1252.

10. Koutinas A, Polizopulou ZS, Saridomichelakis MN, Argyriadis D, Fytianou A, Plevaraki K. 1999. Clinical considerations on canine visceral leishmaniosis in Greece: A retrospective study of 158 cases (1989-1996). J Am Ass Hosp Anim 35: 376-383.

11. Macharia JC, Bourdichon AJ, Gicheru MM. 2004. Efficacy of trypan ${ }^{\circledR}$ : a diminazene based drug as anti-leishmanial agent. Acta Trop 92: 267-272.

12. Melby PC, Yang Y, Cheng J, Zhao W. 1998. Regional differences in the cellular immune response to experimental cutaneous or visceral infection with Leishmania donovani. Infect Immun 66: 18-27.

13. Melby PC, Tryon VV, Chandrasekar B, Freeman GL. 1998. Cloning of syrian hamster (Mesocricetus auratus) cytokine cDNAs and analysis of cytokine mRNA expression in experimental visceral leishmaniasis. Infect Immun 66: 2135-2142.

14. Melby PC, Chandrasekar B, Zhao W, Coe JE. 2001. The hamster as a model of human visceral leishmaniasis: progressive disease and impaired generation of nitric oxide in the face of a prominent Thl-like response. $J$ Immunol 166: 1912-1920.

15. Mojzisova J, Paulik S, Ondrasovic M, Bajova V. 2004. Immune response and immunomodulatory effect of levamisole in immunosupressed dogs vaccinated against parvovirus. Bull Vet Instit Pulawy 48: 5.

16. Restrepo-Salazar JG. 2006. Terapéutica Veterinaria, Ed. Corporación para Investigaciones Biológicas, Medellín, Colombia, p. 154.

17. Sajid MS, Muhammad G, Iqbal MU. 2006. Immunomodulatory effect of various anti-parasitics: a review. Parasitology 132: 13.

18. Spreng D. 1993. Leishmanial polyarthritis in two dogs. $J$ Small Anim Pract 34: 559-563. 